Revue internationale P.M.E.

Économie et gestion de la petite et moyenne entreprise

Revue

internationale

PME

\title{
Rationnement du crédit et PME: une tentative de mise en relation
}

\section{Maria Psillaki}

Volume 8, numéro 3-4, 1995

URI : https://id.erudit.org/iderudit/1008359ar

DOI : https://doi.org/10.7202/1008359ar

Aller au sommaire du numéro

\section{Éditeur(s)}

Presses de l’Université du Québec

ISSN

0776-5436 (imprimé)

1918-9699 (numérique)

Découvrir la revue

\section{Citer cet article}

Psillaki, M. (1995). Rationnement du crédit et PME: une tentative de mise en relation. Revue internationale P.M.E., 8(3-4), 67-90.

https://doi.org/10.7202/1008359ar

\section{Résumé de l'article}

et article part du constat que les petites et moyennes entreprises ont en général un accès au financement externe beaucoup plus difficile que les grandes entreprises. Cela peut s'expliquer par le fait que le risque de défaillance des PME est statistiquement plus élevé que pour les grandes entreprises.

Notre contribution vise à présenter les raisons qui expliquent, sur le plan théorique, le refus de financement pour ce type d'entreprises. Pour ce faire, nous considérons que l'analyse de rationnement de crédit peut nous servir comme support théorique pour expliquer cette exclusion des PME en matière de financement.

Dans cette perspective, cette analyse répond à un double objectif: premièrement, étudier le phénomène du rationnement du crédit et plus particulièrement celui du " redlining " et montrer qu'il permet d'expliquer l'exclusion des PME aux ressources financières ; deuxièmement, proposer des pistes de recherche qui permettent d'envisager une diminution de l'ampleur de celui-ci. Dans cette optique, la prise en compte des relations de long terme et de clientèle constituent un moyen pour réduire le rationnement du crédit et jouent, par conséquent, un rôle fondamental dans l'octroi de prêts aux PME.
Ce document est protégé par la loi sur le droit d'auteur. L'utilisation des services d'Érudit (y compris la reproduction) est assujettie à sa politique d'utilisation que vous pouvez consulter en ligne.

https://apropos.erudit.org/fr/usagers/politique-dutilisation/ 


\title{
Rationnement du crédit et PME: une tentative de mise en relation
}

Maria PSILLAKI*

LATAPSES

MOTS CLÉS

\author{
Asymétrie d'information - Risque de défaut - \\ Rationnement du crédit (redlining) - Relation de clientèle - \\ Équilibre avec séparation des contrats
}

\begin{abstract}
RÉSUMÉ
Cet article part du constat que les petites et moyennes entreprises ont en général un accès au financement externe beaucoup plus difficile que les grandes entreprises. Cela peut s'expliquer par le fait que le risque de défaillance des $P M E$ est statistiquement plus élevé que pour les grandes entreprises.
\end{abstract}

Notre contribution vise à présenter les raisons qui expliquent, sur le plan théorique, le refus de financement pour ce type d'entreprises. Pour ce faire, nous considérons que l'analyse de rationnement de crédit peut nous servir comme support théorique pour expliquer cette exclusion des PME en matière de financement.

Dans cette perspective, cette analyse répond à un double objectif: premièrement, étudier le phénomène du rationnement du crédit et plus particulièrement celui du "redlining " et montrer qu'il permet d'expliquer l'exclusion des PME aux ressources financières; deuxièmement, proposer des pistes de recherche qui permettent d'envisager une diminution de l'ampleur de celui-ci. Dans cette optique, la prise en compte des relations de long terme et de clientèle constituent un moyen pour réduire le rationnement du crédit et jouent, par conséquent, un rôle fondamental dans l'octroi de prêts aux PME.

* Maria Psillaki est titulaire d'un DEA en organisation industrielle et financement de la production et achève actuellement son doctorat en bénéficiant d'une allocation de recherche du ministère de la Recherche et de l'Enseignement au LATAPSES. Elle est aussi chargée d'enseignement de travaux dirigés à l'Université de NiceSophia-Antipolis. Ses principaux domaines de recherche concernent l'étude du fonctionnement du marché du crédit et des titres. Adresse : LATAPSES, 250, rue A.-Einstein, Sophia-Antipolis 1, 06560 Valbonne, France.

L'auteure tient à remercier R. Arena, M. Messori et $\mathrm{D}$. Torre pour les précieuses remarques, critiques et suggestions qu'ils ont bien voulu formuler lors de l'élaboration de cet article. 


\section{ABSTRACT}

The starting point of this article is the observation that, because of statistically higher risk of default, the access to external finance of small and medium sized firms is in general more difficult than in the case of large ones.

The aim of our paper is to offer a theoretical explanation of why such firms are denied finance. Credit rationing, and more specifically redlining, is considered here as offering such an explanation.

In addition, the goal here is to provide some indications of how the credit rationing that small and medium sized firms endure can be reduced. Long term and customer relationships can be considered as a means of reducing credit rationing. They thus play a fundamental role in the process of the granting of loans to such firms.

\section{RESUMEN}

Este articulo parte del hecho de que las pequeñas y medias empresas tienen un acceso al financiamento externo mucho más dificil que las grandes empresas. Esto puede explicarse porque el risgo de cierre de las PyMEs es estaticamento más elevado que para las grandes empresas.

Nuestra contribución tiene por objeto commo se puede teóricamente el recharo financiero en este tipo de empressas. Consideramos que la análisis de racionamiento de credito puede servimos como apoyo teórico para explicar esta exclusion de las PyMEs en materia de financiamiento. Desde esta perspectiva, este análisis responde a un doble objectivo: por un lado estudiar el fenomeno de racionamiento de credito y mas particularmente "redlining" $y$ de enseñar que permite explicar la exlusion de las PyMEs de los recursos financieros, y por otro lado proponer las vias de investicatión que permitén considerar una disminución de su amplitud.

Desde ésta optica, tomar en cuenta las relaciones à largo plazo y de clientela constituyen un medio para reducir el racionemiento del credito y por consecuencia jugarlá un papel fundamental a la bora de conceder préstamos a las PyMEs.

\section{Introduction}

Les petites et moyennes entreprises connaissent des difficultés financières et souffrent d'une insuffisante participation aux modes de financement nouveaux comme le recours direct au marché financier. Faute de pouvoir accéder à ces nouveaux produits, les entreprises de petite et moyenne dimension n'auraient $\mathrm{pu}$, contrairement aux grandes, faire jouer la concurrence entre produits bancaires et produits de marché pour bénéficier d'un alignement des conditions bancaires sur le taux de marché monétaire (Belletante, 1991). 
Au-delà des problèmes de coût du crédit et de structure de financement, le principal handicap des PME tient aux discriminations qu'elles subissent en matière d'accès aux ressources. Souvent, le financement des PME est considéré par les prêteurs comme étant plus dangereux que celui des grandes entreprises. D'après la Centrale des Bilans de la Banque de France (1988), le risque de défaillance décroît avec la taille de l'entreprise. Par conséquent, la traduction du risque dans le financement prend souvent deux formes: celle du rationnement, lequel se traduit par la non-obtention de toutes sortes de crédit pour une entreprise en création ou par le refus de concours supplémentaires pour les entreprises plus anciennes, et celle d'une différenciation des taux débiteurs (Psillaki, 1992).

Ces constats nous conduisent à nous interroger sur le financement des petites et moyennes entreprises; et nous pensons que la littérature qui traite des asymétries d'informations entre prêteurs et emprunteurs au marché du crédit permet d'expliquer l'éviction que subissent ces entreprises en matière de financement. Plus particulièrement, nous pensons que l'analyse de rationnement du crédit peut nous servir comme support théorique pour justifier le refus de financement de ce type d'entreprises.

La présente contribution a un double objectif: d'une part, montrer qu'effectivement l'analyse du rationnement du crédit (redlining) est en mesure d'expliquer l'exclusion des PME aux ressources financières et, d'autre part, de proposer des pistes de recherche qui permettent d'envisager une diminution de celui-ci. Pour ce faire, nous avançons que les relations de clientèle et de long terme diminuent les comportements opportunistes et les incitations, améliorant ainsi la probabilité de réussite de l'emprunteur.

Contrairement aux marchés de biens classiques, le marché du crédit est particulier, et ce, dans la mesure où l'octroi d'un prêt peut s'assimiler à la vente d'un bien à un prix aléatoire. Ainsi, lorsque le taux d'intérêt est fixé au moment de la décision d'octroi, il subsiste un risque de non-paiement des intérêts et du principal lors des échéances futures. En outre, le marché du crédit est caractérisé par une asymétrie d'information entre les offreurs et les demandeurs, asymétrie qui est à la base de l'explication du phénomène de rationnement du crédit, c'est-à-dire de l'exclusion de certains emprunteurs potentiels aux fonds prêtables (Stiglitz et Weiss, 1981).

Toutefois, l'activité de crédit associe intimement deux notions, celle de la confiance et celle du temps. Par la suite, en introduisant ainsi les relations de long terme et de clientèle (Okun, 1981; Sharpe, 1990), nous essayerons de montrer qu'elles permettent de réduire le rationnement du crédit et qu'elles jouent un rôle fondamental dans l'octroi de prêts aux PME (Dietsch, 1989). 


\section{Le risque d'insolvabilité comme facteur d'exclusion des PME aux fonds prêtables}

La relation de prêt associe des agents ayant des intérêts distincts. L'emprunteur est concerné par la rentabilité des capitaux empruntés, tandis que le prêteur l'est par la solvabilité du premier. De plus, le marché du crédit est caractérisé par une asymétrie d'information entre les agents. Dès lors que l'imperfection de l'information est prise en compte, il devient impossible pour le prêteur de déterminer la qualité de l'emprunteur (la qualité du projet financé), ou impossible ou très coûteux de surveiller les actions d'un agent. En effet, l'imperfection de l'information à laquelle font face les prêteurs exerce une influence considérable sur l'évaluation de la qualité des emprunteurs potentiels présents sur le marché du crédit conformément à l'approche développée par Akerlof (1970). Par conséquent, à partir du moment où les effets de sélection adverse (changement de la qualité moyenne des demandeurs) et de risque moral (les demandeurs entreprennent des projets plus risqués) sont présents sur le marché du crédit, les changements de prix affectent la qualité moyenne des projets d'investissement des emprunteurs (Stiglitz, 1987; Stiglitz et Weiss, 1981; Levratto, 1992).

En effet, en matière de décision d'octroi de crédit, le problème de sélection adverse ${ }^{1}$ est essentiel. Entre une banque et ses clients existe une asymétrie d'information initiale relative, par exemple, au risque du projet à financer. Dans ce cas, les actions prises par les agents les moins informés peuvent avoir des effets non désirés, dès lors qu'elles peuvent affecter de façon contraire (adverse) la qualité des emprunteurs potentiels (corrélation entre la qualité des emprunteurs et le prix du marché, ce qui fait que tout changement de taux d'intérêt affecte la probabilité de défaillance de l'emprunteur et cela peut empêcher le marché de se solder). Tous ces effets impliquent l'existence d'un taux d'intérêt «optimal " pour la banque qui peut être "visqueux», voire rigide, même dans le cas d'un excès de demande.

Toutefois, nous considérons que comparativement aux grandes entreprises, les PME présentent des caractéristiques qui leur sont propres et qui ne font qu'accroître l'effet de sélection adverse. Celles-ci sont liées au fait que la durée de vie est beaucoup plus brève chez la PME, que ce sont souvent des entreprises sous-capitalisées, qui présentent une structure interne différente de celle des

1. La sélection adverse dans le cas du marché du crédit se réfère à des situations où des emprunteurs de «qualité » variable sont présents sur le marché : l'une des deux parties du marché peut observer la qualité des emprunteurs, l'autre partie a une certaine connaissance statistique concernant la qualité des emprunteurs, comme la fonction de distribution de probabilité de rendement de l'investissement effectué par l'emprunteur. 
grandes entreprises et, finalement, qui ont souvent un poids faible dans l'espace concurrentiel. Dans un premier temps, examinons analytiquement ces points interdépendants qui expliquent leur fragilité et, par conséquent, leur risque de défaillance. Ensuite, nous verrons comment se traduit théoriquement le risque dans le financement des PME.

\subsection{Spécificités des PME et risque de défaillance}

Malgré le fait que les PME présentent un taux de «natalité» supérieur à celui des grandes entreprises, elles affichent en même temps un taux de "mortalité» aussi élevé. Cela explique la réticence des banques en ce qui concerne le financement aux PME, puisque le risque de non-remboursement d'un crédit à moyen et long terme est grand, étant donné qu'une PME peut disparaître en quelques semaines sous les coups d'une crise de trésorerie, par exemple.

En outre, l'insuffisance des fonds propres des PME est souvent invoquée comme une des principales sources de fragilité de ces entreprises. Toutefois, ce facteur leur permet d'obtenir des rentabilités supérieures, ou du moins comparables, à celles des firmes plus grandes et de bénéficier d'un effet de levier positif (Paranque, 1992). Cependant, l'étendue de leur endettement les fragilise du fait des prélèvements financiers qu'il requiert, compte tenu des taux d'intérêts plus élevés assumés en général par les PME.

Nous avons mentionné que la structure interne et l'organisation des PME peut être un facteur favorable à l'augmentation de la sélection adverse. Souvent le cycle de décision stratégique, où l'horizon temporel de la PME ne répond pas à une politique de long terme, est axé sur la réaction plutôt que sur l'anticipation. Selon Julien (1994, p. 279), ces entreprises emploient peu de méthodes et de techniques de gestion telles que la prévision, l'analyse financière et la gestion de projet.

Le recours au crédit se pose alors pour la PME à chaque fois comme une décision isolée et non comme un élément d'une politique de financement courant de l'entreprise défini à l'avance.

Les PME présentent donc une double spécificité: une sensibilité trop forte à l'environnement (investissement mal ciblé, par exemple) et une possibilité de rentabilité qui rend le responsable de l'entreprise moins sensible au coût du financement (effet de hasard moral) de son investissement. Si le projet réussit, sa rentabilité lui permettra de rembourser ses échéances. Dans le cas contraire, la liquidation judiciaire de la PME pourra libérer l'entrepreneur de la charge des remboursements, créant une certaine indifférence à l'égard du poids de la charge financière. 
Les ressources limitées des PME ainsi que leur mode de gestion propre axé généralement sur le non-structuré, sur le court terme et sur la réaction plutôt que sur la prévision imposent des contraintes spécifiques que les prêteurs doivent prendre en compte. Ces faiblesses justifient le fait que les PME ont un taux de mortalité beaucoup plus élevé que les grandes entreprises, résultant à la fois de facteurs externes (liés à l'environnement ou à la conjoncture) et de facteurs internes.

\subsection{Risque de défaillance et rationnement du crédit}

De ce qui précède, il apparaît que l'incertitude présente dans toute relation de crédit, et justifiée par la reconnaissance de la dimension temporelle caractérisant cette relation, porte sur les états de la nature, sur la qualité de la demande de l'entrepreneur, mais aussi sur son comportement dans le futur. Ce qui fait que sur ce marché spécifique, le taux d'intérêt n'a pas la capacité pour synthétiser l'information et solder le marché - capacité que possède le prix sur le marché canonique (Stiglitz et Weiss, 1981).

Stiglitz et Weiss montrent que dans la mesure où le taux d'intérêt est en relation avec le risque du groupe des emprunteurs, lequel peut ne pas être connu des banques, il est possible d'aboutir à l'obtention d'équilibres avec rationnement des emprunteurs potentiels. En effet, les banques ne souhaitent pas satisfaire certains clients potentiels prêts à contracter à un taux d'intérêt supérieur à celui qu'elles annoncent, car cette disposition est perçue comme étant révélatrice d'emprunteurs à haut risque de défaut, qu'il faut alors écarter par rationnement.

Le choix de ce modèle s'explique par le fait que dans l'analyse de Stiglitz et Weiss (1981), le seul moyen de financement des entreprises est celui du crédit bancaire (c'est-à-dire qu'il n'y a pas d'arbitrage entre financement externe et financement interne). Cette hypothèse correspond bien au type d'entreprises que nous étudions: les firmes de petite et moyenne taille. Or, on sait que le crédit bancaire occupe une place importante dans l'endettement des PME (Yoncourt et Marois, 1990).

Par conséquent, lorsque la banque décide du montant de financement accordé à un emprunteur, elle doit tenir compte du fait que les emprunteurs ne sont pas tous de même «qualité» (le risque d'insolvabilité n'est pas le même pour tous les emprunteurs) et que tout changement du taux d'intérêt aura une conséquence sur la qualité de la demande. Dans ce cas, les pressions endogènes qui poussent le prix à l'équilibre walrasien stable peuvent ne pas exister et il peut y avoir des prix différents de ceux qui soldent le marché (Stiglitz et Weiss, 1981 ; 1983). Ainsi, l'équilibre peut se caractériser par une multiplicité de prix. 
En effet, le prêteur peut proposer différents taux d'intérêts selon la période considérée ou selon la solvabilité de l'emprunteur.

Stiglitz et Weiss (1981) considèrent deux types de firmes présentes sur le marché: les firmes risquées $h$, et les firmes peu risquées (Mattesini, 1993). Les auteurs font l'hypothèse que les prêteurs sont incapables de distinguer les deux types de firmes. Si l'on note par $h$ le nombre des firmes risquées et par $v$ les firmes peu risquées, le taux de rendement moyen de la banque sera:

$$
\bar{\rho}(r)=\frac{\mu_{h} \rho_{h}(r)+\mu_{v} \rho_{v}(r)}{\mu_{h}+\mu_{v}}
$$

Grâce à cette équation, nous pouvons constater que le taux de rendement augmente lorsque le taux d'intérêt augmente. Toutefois, si le taux d'intérêt augmente au-delà du taux d'intérêt de réservation, les firmes les moins risquées sortiront du marché et, par conséquent, cela diminuera le taux moyen de rendement. Donc, si cet effet négatif de taux d'intérêt l'emporte sur l'effet positif, le rendement de la banque diminuera et cette fonction ne sera pas une fonction monotone du taux d'intérêt (effet de sélection adverse). Une autre raison explique pourquoi le taux de rendement n'est pas une fonction monotone du taux d'intérêt: c'est la présence de l'effet de hasard moral. Effectivement, lorsque le taux d'intérêt augmente, les emprunteurs sont incités à abandonner les projets les moins risqués pour entreprendre des projets plus risqués afin de compenser la diminution des recettes due à l'accroissement du taux d'intérêt. Dès lors que l'on considère l'offre de crédit en tant que fonction croissante du taux de rendement $\left(S()\right.$, avec $\left.\frac{\partial S}{\partial \bar{r}}>0\right)$ et étant donné que ce taux commence à décroître au-delà d'un certain niveau, il sera optimal pour la banque (sous la condition d'information imparfaite) de ne pas accroître le taux d'intérêt mais plutôt de rationner le crédit, afin de pouvoir garder sur le marché les firmes les moins risquées. L'analyse faite par Stiglitz et Weiss ne nécessite pas le recours aux facteurs institutionnels ou aux régulations gouvernementales pour expliquer le rationnement du crédit. Ces auteurs essaient d'expliquer pourquoi certaines personnes ne peuvent emprunter des fonds à n'importe quel taux d'intérêt, lorsque des individus avec des caractéristiques similaires ont accès aux fonds prêtables.

Or, d'après la Centrale des Bilans de la Banque de France (1988), il semble que le risque de défaillance d'une entreprise décroît à mesure que la taille de l'entreprise augmente (tableau 1). 
TABLEAU 1

Le risque de défaillance est plus élevé pour les PME

\begin{tabular}{|c|c|c|c|c|c|c|c|}
\hline & \multirow[b]{2}{*}{ Classes de risques } & \multicolumn{3}{|c|}{ PME } & \multicolumn{3}{|c|}{ Grandes entreprises } \\
\hline & & 1987 & 1988 & 1989 & 1987 & 1988 & 1989 \\
\hline Défavorable & $\begin{aligned} Z & <-1,875 \\
-1,875 \leq Z & <-0,875 \\
-0,875 \leq Z & <-0,25\end{aligned}$ & $\begin{array}{l}4,2 \\
4,9 \\
5,9\end{array}$ & $\begin{array}{l}4,1 \\
4,3 \\
5,9\end{array}$ & $\begin{array}{l}3,0 \\
4,0 \\
5,7\end{array}$ & $\begin{array}{l}3,3 \\
5,0 \\
3,7\end{array}$ & $\begin{array}{l}2,5 \\
4,7 \\
3,9\end{array}$ & $\begin{array}{l}\text { n.s. } \\
\text { n.s. } \\
\text { n.s. }\end{array}$ \\
\hline Neutre & $0,25 \leq \mathrm{Z}<-0,125$ & 8,5 & 7,6 & 7,4 & 3,0 & 3,6 & n.s. \\
\hline Favorable & $\begin{array}{l}0,125 \leq Z<-0,625 \\
0,625 \leq Z<-1,25 \\
1,25 \leq Z<\end{array}$ & $\begin{array}{l}18,9 \\
29,3 \\
28,3\end{array}$ & $\begin{array}{l}18,8 \\
31,1 \\
28,2\end{array}$ & $\begin{array}{l}20,2 \\
30,5 \\
29,2\end{array}$ & $\begin{array}{l}13,5 \\
42,3 \\
29,2\end{array}$ & $\begin{array}{l}12,0 \\
42,0 \\
31,3\end{array}$ & $\begin{array}{l}\text { n.s. } \\
\text { n.s. } \\
\text { n.s. }\end{array}$ \\
\hline
\end{tabular}

Source : Centrale des Bilans de la Banque de France.

Le risque de défaillance des PME est plus élevé que celui des grandes entreprises : 14,3\% des PME avaient en 1988 un «score $Z^{2}$ » défavorable contre seulement $11,1 \%$ des grandes entreprises. En effet, le risque de défaillance décroît avec l'augmentation de la taille de l'entreprise; et si $22,6 \%$ des entreprises de moins de 20 salariés ont un «score» défavorable, seulement $17 \%$ des entreprises de 20 à 100 salariés et $12,3 \%$ des entreprises de 400 à 500 salariés sont dans ce cas (Roger-Machart, 1991, p. 135).

Cette relation inverse entre la taille de l'entreprise et le risque qu'elle présente se confirme pour les années suivantes. En effet, d'après le tableau suivant, le taux de défaillance ${ }^{3}$ des entreprises en 1992 est de $3,9 \%$ pour les PME contre seulement $0,7 \%$ pour les grandes entreprises (soit environ cinq fois moins).

Comme nous le constatons par ce graphique à l'intérieur des PME, ce taux est inversement lié à la taille des entreprises: il passe de $2,3 \%$ pour les entreprises de 100 à 500 salariés à $4,4 \%$ pour celles qui emploient entre 10 et 20 salariés.

Ainsi, nous pensons que la deuxième forme de rationnement donnée par Stiglitz et Weiss permettrait d'illustrer l'exclusion des PME du marché du crédit. En effet, selon Stiglitz et Weiss (1981), si l'on divise la population des

2. La fonction « $\mathrm{Z}$ » est une combinaison linéaire de plusieurs ratios économicofinanciers (frais financiers sur résultat économique brut, couverture des capitaux investis, delais crédit-fournisseurs, etc.) qui permet de distinguer les entreprises en difficulté des entreprises saines (Bardos, 1991).

3. Le taux de défaillance se définit comme le nombre des entreprises défaillantes par rapport au nombre des entreprises vivantes. 
emprunteurs potentiels en plusieurs groupes d'individus identiques, chaque groupe se différenciant des autres selon un critère de risque, il est possible d'observer qu'un ou plusieurs groupes d'individus identiques sont exclus totalement du marché du crédit par les prêteurs (redlining).

GRAPHIQUE 1

Taux de défaillance des entreprises en 1992

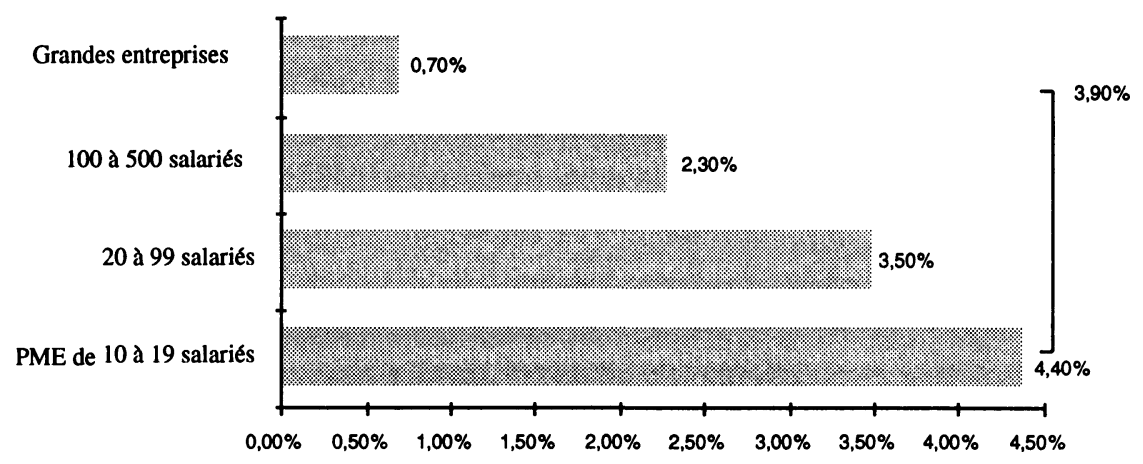

Source : Sofaris, AFB (1994).

Stiglitz et Weiss, dans leur modèle de 1981, ont avancé qu'à l'équilibre, le marché du crédit peut ne pas nécessairement se caractériser par un prix unique, mais par une distribution de prix. En effet, le rendement espéré ne présente pas un maximum global unique par rapport au taux d'intérêt. Ainsi, d'après la proposition 6 de Stiglitz et Weiss, si l'espérance moyenne de revenu $\bar{\rho}$ sur un prêt a plusieurs modes, c'est-à-dire qu'il existe plusieurs maximums locaux (graphique 2), alors dans ce cas (comme on l'a vu précédemment, l'espérance moyenne de revenu n'était pas une fonction monotone du taux d'intérêt), l'équilibre de marché peut se caractériser soit par un taux d'intérêt unique égal ou inférieur au taux qui solde le marché $r_{\mathrm{m}}$, soit par deux taux d'intérêt avec excès de demande de crédit pour le plus faible.

Ainsi qu'il apparaît sur le graphique, il existe deux valeurs de taux d'intérêt, $r_{1}$ et $r_{2}$, qui rapportent à la banque le même rendement espéré par prêt. Supposons qu'il n'existe qu'un taux d'intérêt $r_{1}$, alors à ce taux est associé un excédent de demande de prêts. D'un autre côté, supposons que seul $r_{2}$ soit proposé; dans ce cas, il y aurait un excès d'offre. Or, il existe une distribution de fonds prêtables disponibles aux emprunteurs telle que les emprunteurs qui n'ont pas pu emprunter au taux d'intérêt $r_{1}$ empruntent au taux d'intérêt $r_{2}$. Toutefois, les emprunteurs dont le taux de réservation se trouve entre $r_{1}$ et $r_{2}$ seront rationnés. 
GRAPHIQUE 2

Équilibre avec deux valeurs du taux d'intérêt

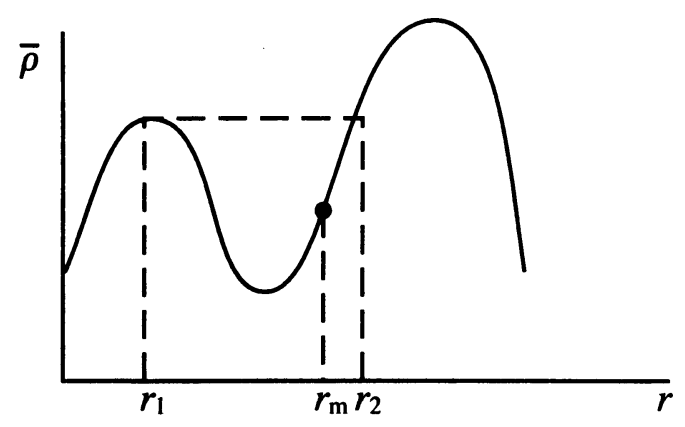

Source : Stiglitz, J.E. et A. Weiss (1981), « Credit rationing in markets with imperfect information », p. 398.

En conséquence, cette proposition (6) nous permet d'introduire la seconde forme de rationnement. Elle correspond à la situation où un ou plusieurs groupes d'individus identiques sont exclus totalement du marché du crédit par les prêteurs. Considérons trois groupes d'individus identiques auxquels la banque facturera les taux d'intérêt optimaux $r_{1}, r_{2}$, et $r_{3}$. À ces taux correspondent les revenus attendus par la banque sur ces prêts consentis $\rho_{1}, \rho_{2}$ et $\rho_{3}$. Stiglitz et Weiss en déduisent que les taux d'équilibre sont tels que si les trois groupes d'individus reçoivent du crédit, alors $\rho_{1}, \rho_{2}$ et $\rho_{3}$ sont égaux (théorème 14, p. 406). En outre, si E' représente le coût des fonds de la banque (coût de refinancement de la banque), la concurrence bancaire sera telle que les profits seront nuls, soit finalement:

$$
\rho_{1}=\rho_{2}=\rho_{3}=\mathrm{E}^{\prime}
$$

Ces résultats sont illustrés sur le graphique suivant (graphique 3).

$\mathrm{Si}$, par contre, le coût des fonds de la banque est $\mathrm{E}^{\prime \prime}$ tel que $\mathrm{E}^{\prime \prime}>\mathrm{E}^{\prime}$, alors aucun des individus du groupe 1 ne pourra obtenir de crédit: quel que soit le taux d'intérêt qui peut être servi à ce groupe, la rentabilité obtenue par la banque est inférieure au coût des fonds prêtés. Toutefois, la banque servira tous les individus du groupe 3 à un taux $r_{3}^{\prime}$; les individus du groupe 2 se verront offrir du crédit au taux $r_{2}^{\prime}$ dans la limite des disponibilités de la banque. Ainsi, un groupe entier d'individus est exclu du marché du crédit (le groupe 1), marché dans lequel certains individus du groupe 2 sont susceptibles de ne pas recevoir de crédit. 


\section{GRAPHIQUE 3}

\section{Rationnement du crédit dans le cas de plusieurs groupes homogènes}

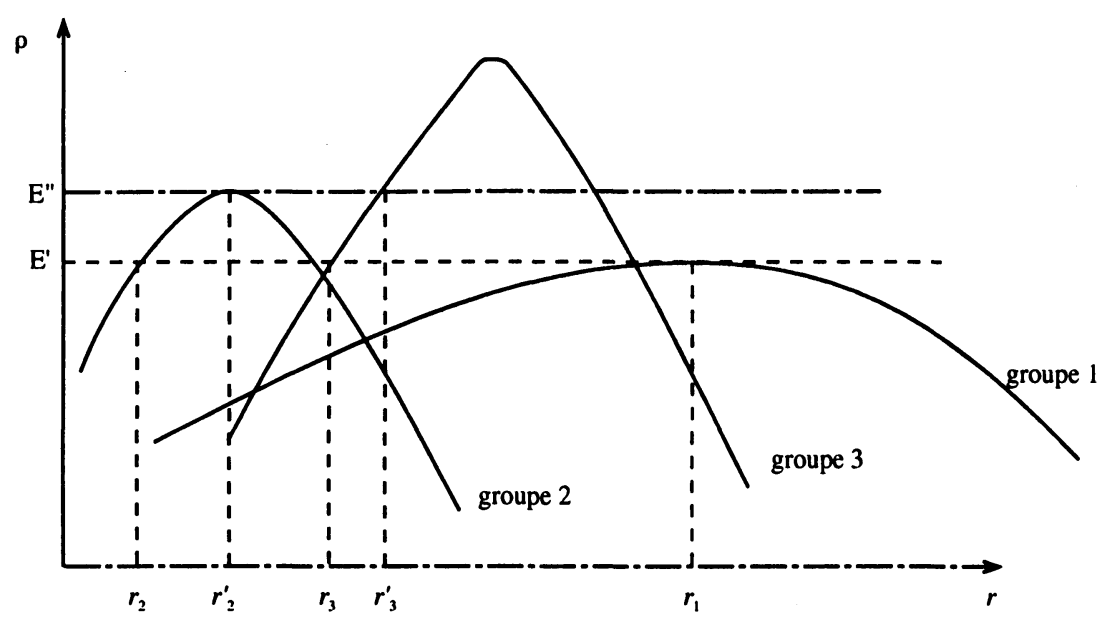

Source: Stiglitz, J.E. et A. Weiss (1981), ibid., p. 406.

Même lorsque l'on considère qu'il y a plusieurs ensembles différents d'emprunteurs et que le rationnement du crédit peut ne pas être un phénomène significatif (Riley, 1987), cette hypothèse, comme Stiglitz et Weiss (1987) l'ont fait remarquer dans leur réponse à Riley, ne remet pas en cause la seconde forme de rationnement.

Donc, cette forme de rationnement permet d'expliquer le refus de financement aux PME, puisqu'elles sont souvent considérées par les prêteurs comme des entreprises trop risquées.

Certes, dans leurs taux de prêt aux PME, les banques répercutent une marge de risque additionnel. Mais une étude de Sofaris (AFB, 1994) montre que, depuis quatre ans, ces marges sont devenues nettement insuffisantes pour faire face aux pertes supplémentaires occasionnées par les crédits. On peut constater ce déséquilibre à l'aide du graphique 4 .

Par conséquent, il nous semble que l'analyse faite par Stiglitz et Weiss (1981) sur la notion et la forme de rationnement trouve une application directe dans le cas des PME où, pour certaines d'entre elles, le coût de refinancement de la banque est supérieur au rendement attendu. 
Toutefois, l'analyse de Stiglitz et Weiss est basée sur l'hypothèse que tous les emprunteurs potentiels demandent le même montant de financement. Or, Milde et Riley (1988) supposent, contrairement à Stiglitz et Weiss (1981), que la demande de financement est variable et que cela représente un moyen pour les emprunteurs de signaler leur qualité auprès des banques. En effet, la taille de l'emprunt peut constituer un moyen pour identifier les différences de qualité des projets. Par conséquent, considérer que le montant de financement demandé est variable constituera un signal pour la banque sur le type de l'emprunteur. Selon ces deux auteurs, les emprunteurs qui ont des projets moins risqués préfèrent des emprunts plus élevés. Ainsi, la valeur du prêt demandé par l'emprunteur constitue un signal pour la banque sur la qualité de ce dernier.

GRAPHIQUe 4

Encours des créances douteuses

(en milliards de francs)

et ratio créances douteuses / encours de crédits bruts

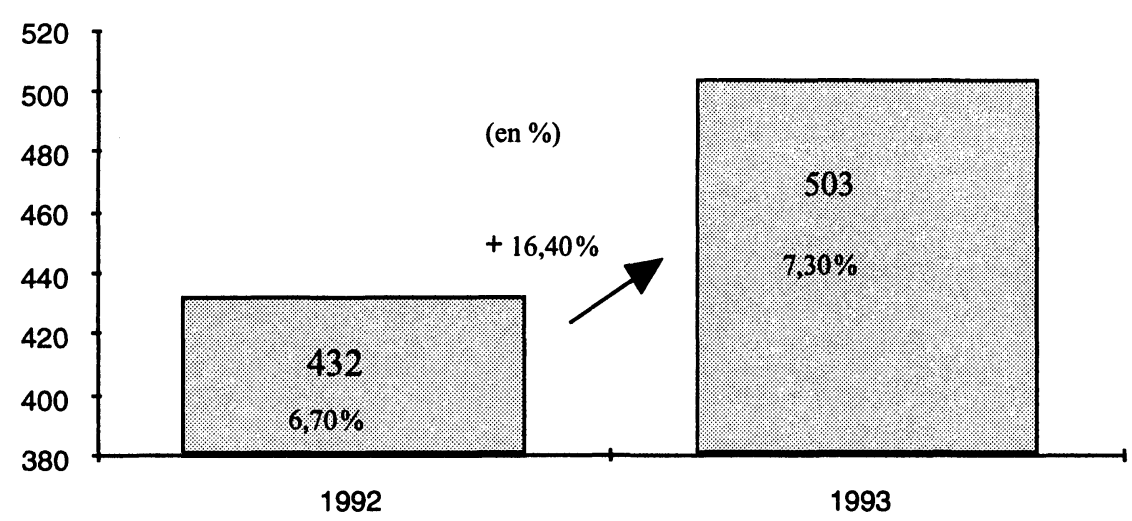

Source : Rapport de la Commission bancaire.

Donc, dès lors que nous considérons la taille de la demande de financement comme variable, nous admettons que les banques peuvent offrir des prêts de taille différente à des taux d'intérêt différents. En conséquence, par les différences de taux marginal de substitution des emprunteurs, la banque peut discriminer les différentes classes de risque. Ainsi, l'équilibre est un équilibre avec séparation des contrats sans rationnement et non un équilibre avec agrégation des contrats avec rationnement, comme celui de Stiglitz et Weiss. En effet, si la banque est capable de distinguer les emprunteurs à l'équilibre, il 
y aura plusieurs contrats offerts. Nous pouvons alors présenter sur un graphique les deux contrats offerts par la banque pour les deux types d'emprunteurs. Les emprunteurs dont le projet d'investissement est de bonne qualité $\theta_{1}$ et ceux de moins bonne qualité $\theta_{1}$, avec donc $\theta_{2}>\theta_{1}$ où $\theta$ est un paramètre de qualité.

Sur le graphique suivant, d'une part, l'axe des abscisses représente le montant de financement (L) et l'axe des ordonnées représente $R=1+r$, où $r$ est le taux d'intérêt servi par la banque pour un montant de financement $L$. D'autre part, sur l'axe des ordonnées figure également $i$, défini comme le coût d'opportunité des fonds prêtables. Enfin, $\mathrm{II}_{1}(\mathrm{~L}, \mathrm{R})$ et $\mathrm{II}_{2}(\mathrm{~L}, \mathrm{R})$ représentent les courbes d'iso-profit de la banque et représentent les courbes d'iso-utilité des emprunteurs.

GRAPHIQUe 5

Équilibre du marché du crédit lorsque la demande de financement est variable

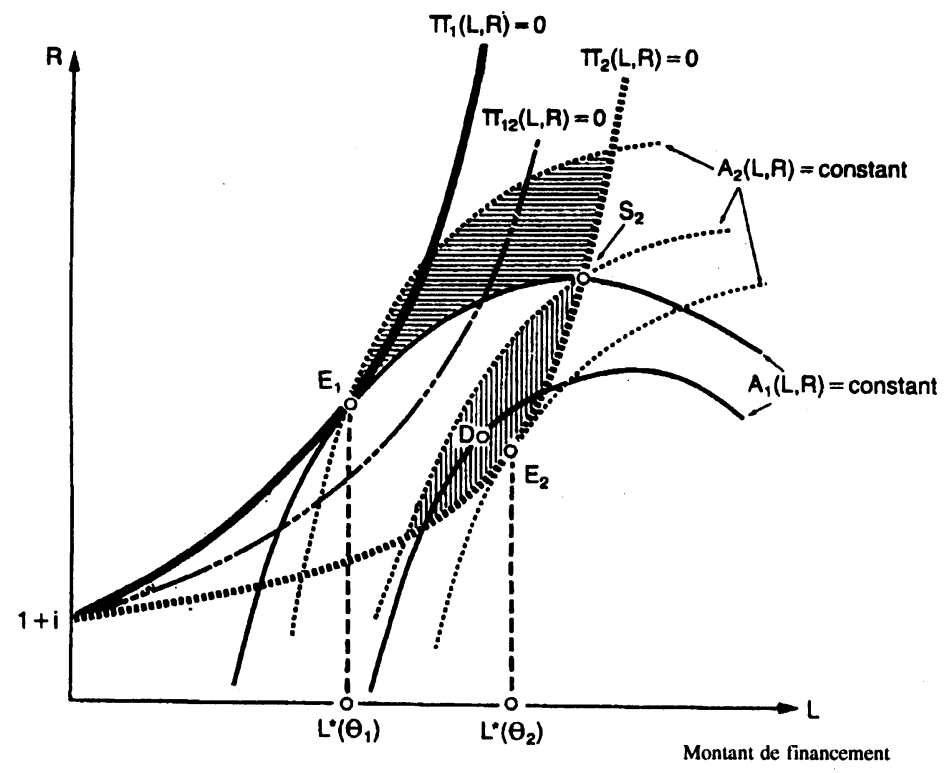

Source: Milde, H. et J.G Riley (1988), «Signaling in credit markets», The Quarterly Journal of Economics, vol. CIII, $\mathrm{n}^{\circ} 1$, février, p. 108. 
Ce graphique permet de constater que le contrat $S_{2}$ procure des gains plus élevés pour les «bons» emprunteurs, tandis que le contrat $\mathrm{E}_{1}$ est strictement préféré à tout autre contrat par les «mauvais» emprunteurs. En outre, l'équilibre avec agrégation des contrats (point $\mathrm{D}$ ) est privilégié par tous les emprunteurs mais puisqu'il n'y a pas de point d'intersection avec la courbe de profit nul de la banque, cette éventualité ne peut être retenue.

Toutefois, si l'information concernant la qualité et l'effort des emprunteurs est asymétrique, l'ensemble de ces contrats peut ne pas constituer un équilibre, puisque les moins bons emprunteurs peuvent demander les mêmes contrats que ceux demandés par les bons emprunteurs. Par conséquent, un équilibre avec séparation de contrats nécessite que les contrats des bons emprunteurs ne se trouvent pas au-dessous de la courbe d'iso-profit des moins bons emprunteurs.

Donc, d'après l'analyse faite par Milde et Riley (1988), la demande de financement peut constituer un moyen pour les banques de dépister les emprunteurs à risque. Par la suite, nous verrons que la source d'information provenant de la demande de financement peut être complétée par les relations de clientèle.

Parvenu à ce niveau, il est bon de distinguer les deux méthodes d'évaluation de l'emprunteur dont le prêteur dispose. La première fait référence à la probabilité que présente chaque entreprise selon les méthodes statistiques (scoring), c'est-à-dire que le prêteur qualifie le risque de l'entreprise selon des critères plutôt objectifs. Cette analyse repose sur la loi des grands nombres et est appliquée indifféremment à tout emprunteur. La deuxième fait plutôt référence à des critères subjectifs. Chaque emprunteur est considéré par le prêteur comme un cas unique. Dans ce cas, le prêteur peut toujours avoir recours à des évaluations comptables, mais selon des méthodes qui lui sont propres. Cette deuxième méthode est basée sur une relation de long terme et de confiance qui permet de réduire les comportements opportunistes en dépit d'une certaine réversibilité que représente la première méthode.

Dans ce cas, prêteur et emprunteur s'engagent dans un processus mutuel de compréhension et de connaissance pour mieux appréhender les besoins de chacun. Dans cette optique, la banque peut offrir des produits non standardisés résultant de l'acquisition et de traitement de l'information sur l'entreprise. Nous parlerons alors de l'établissement d'une relation «personnalisée» qui permettrait à la banque de connaître son client (Rivaud-Danset et Salais 1992, p. 90; Rivaud-Danset, 1995), ce qui donnerait des contrats spécifiques pour chaque entreprise. Ainsi l'équilibre qui résulterait serait un équilibre avec séparation des contrats (separating equilibrium) selon l'idiosyncrasie de l'entreprise considérée. 


\section{Les relations de clientèle comme moyen de réduction du rationnement du crédit aux PME}

Dans l'analyse qui précède, la banque se base uniquement sur une information standardisée sur le client en termes de calcul de probabilité de réussite et exclut, par conséquent, toute compréhension personnalisée. La fidélité et la relation de clientèle au sens d'Okun (1981), comme nous le verrons par la suite, ainsi que la connaissance mutuelle et la participation aux choix de l'entreprise permettent de surmonter l'incertitude inhérente à toute opération de prêt. Lorsque la banque fonde son jugement sur des données objectives (scoring), elle ne distingue pas les situations passagères de défaut de paiement (manque de liquidité) des situations de dépôt de bilan (situations d'insolvabilité). En cas de difficulté de l'entreprise, la banque cesse ses relations avec elle, tandis que lorsque la banque entretient des relations de long terme d'engagement et de confiance, ces relations empêcheront la banque d'interrompre son financement. Il est possible, par ces relations de clientèle et de long terme, d'isoler les différentes étapes qui conduisent une petite firme du défaut de paiement au dépôt de bilan et d'éviter ce dernier.

Pour Okun (1981), en raison des coûts d' «évaluation ${ }^{4}$ » de la performance, les firmes de petite taille ont recours aux banques commerciales et régionales pour se financer. Selón l'auteur, la relation de crédit est fondée sur d'autres principes que celui de la flexibilité des taux débiteurs. Ainsi, dans ces relations, la banque évite à l'emprunteur les trop grandes fluctuations de taux d'intérêt et en même temps, la banque assure ces clients, en cas de restriction monétaire, de les financer au détriment des nouveaux emprunteurs (p. 194). Par conséquent, ce qui joue un rôle central dans cette approche est la connaissance mutuelle des deux acteurs.

Dans ce qui suit, nous montrerons que les relations de clientèle permettent aux banques de mieux évaluer le risque des emprunteurs. Elles contribuent donc à diminuer le rationnement du crédit. Pour le long terme, les banques ont aussi intérêt à offrir des contrats de crédit s'adaptant à la situation des emprunteurs plutôt que des contrats de dettes standard, rigides, ne prenant pas en compte la dimension temporelle et dynamique de la relation de crédit. Toutefois, comme nous le verrons également par la suite, ces relations de clientèle donnent naissance à une information spécifique. Cela implique une rente informationnelle se traduisant par un avantage concurrentiel et un pouvoir de marché des banques sur leurs anciens clients.

4. Traduction du mot « rating ». 


\subsection{Constatations théoriques des relations de clientèle et validations empiriques auprès des PME}

Les modèles théoriques récents traitant des relations de clientèle dans la banque sont pratiquement tous des modèles d'asymétrie d'information (Diamond, 1984 ; Haubrich, 1989; Gale et Hellwig, 1985; Sharpe, 1990; Webb, 1992). Les relations de clientèle permettent, d'une part, d'alléger les coûts d'informations externes et, d'autre part, sont sources de production d'informations internes, ce qui permet de réduire l'asymétrie d'information inhérente à la relation de financement. Cette information peut concerner soit l'entrepreneur, soit son projet. Les informations sur l'entrepreneur peuvent porter sur sa qualification, ses expériences professionnelles, ses capitaux propres, etc. Quant aux informations sur le projet, elles sont à caractère économique et permettent d'en évaluer la qualité sur le plan de l'investissement.

Ainsi, dans le cas d'une asymétrie d'information ex ante (effet de sélection adverse) ou ex post (effet de risque moral), les relations de clientèle permettent, d'une part, de connaître la véritable nature de l'entrepreneur et, d'autre part, de diminuer les coûts liés à la vérification du résultat de l'investissement.

Ces relations de clientèle sont aussi un moyen d'inciter les emprunteurs à révéler leur information, puisque dans le cas contraire, celui où l'emprunteur dissimule l'information, ce dernier risque de voir ses prêts non renouvelés.

C'est précisément la crainte d'un rationnement futur et d'une fin de relation de clientèle avec la banque qui réduit les comportements de hasard moral de l'emprunteur et, de ce fait, limite la probabilité de rationnement de ce dernier. Dans cette perspective, Stiglitz et Weiss (1983) montrent les effets incitateurs de la menace faite par la banque de refuser un crédit pour les périodes suivantes en cas de non-remboursement. Donc, dans une perspective dynamique (analyse multipériodale) et dans le cadre d'une analyse détaillée de la structure organisationnelle des prêteurs et des emprunteurs, les relations de long terme peuvent constituer un moyen pour réduire les effets de sélection adverse et de risque moral de l'emprunteur. Plutôt que d'augmenter le taux d'intérêt sur le crédit, les banques choisissent donc comme stratégie de représailles de ne plus accorder de prêts aux entreprises défaillantes. Ainsi, ce qui est nécessaire pour avoir un équilibre est ce que Stiglitz et Weiss appellent la «crédibilité». Cela signifie que la banque doit toujours honorer son engagement. En conséquence, il peut exister un équilibre dans lequel il y a cessation des relations entre la banque et l'emprunteur pour les agents qui ont fait défaut. L'état d'équilibre nécessite aussi que les entreprises défaillantes ne puissent obtenir de prêt dans aucune autre banque (car cela constitue un mauvais signal, donc aucune autre banque ne leur offrira un contrat). Par conséquent, toute entreprise défaillante ne sera pas financée. 
Ces résultats théoriques de Stiglitz et Weiss (1983) se confirment sur le plan empirique d'après une étude auprès d'un échantillon de PME (Bloch, Bourdieu, Colin-Sedillot et Longueville, 1995). D'après cette étude, les premiers indices de la difficulté financière de l'entreprise modifient la perception des banquiers sur la solvabilité de l'entrepreneur et suscite un ensemble de réactions de leur part: durcissement des conditions, refus de crédit, fermeture du compte, etc. (p. 235).

Dans cette optique, pour Cukierman (1978), le fait que les emprunteurs ont une demande jointe de services bancaires est un élément incitatif pour les banques, ce qui permet de diminuer le niveau de rationnement à ce type d'emprunteurs. D'après cet auteur, la banque peut accepter une baisse de taux d'intérêt servi aux emprunteurs dans la mesure où ces derniers ont une demande considérable de services (tenue de comptes, dépôts à vue, etc.). Par ces services, la banque est en mesure de connaître les partenaires de l'entreprise, ses clients et ses fournisseurs, ce qui est important dans le cas de petites et moyennes entreprises. Par conséquent, selon cette approche, les demandeurs ne seront pas rationnés et obtiendront du crédit à de meilleures conditions.

Sharpe, en 1990, a développé une approche originale des relations de clientèle qu'il considère comme la conséquence d'une asymétrie d'information ex post entre les banques; il essaye alors de déterminer les effets de relations de clientèle en termes d'efficience de l'allocation des ressources. Dans cette optique, seule la banque ayant financé l'entreprise peut observer le résultat du projet de l'emprunteur. Les autres banques n'ont accès qu'à une information, un signal sur ce résultat. Ces banques «externes ${ }^{5}$ » n'observent donc qu'un signal imparfait du résultat des firmes avec lesquelles elles ne sont pas liées. La banque ayant accordé le crédit en première période possède plus d'informations que les autres banques sur son client. Les relations de clientèle donnent naissance à une «information interne»; elles permettent à la banque concernée de mieux connaître l'entreprise financée et, par conséquent, d'évaluer les perspectives d'avenir de son client avec plus de fiabilité. Cette asymétrie d'information entre les banques concurrentes crée un pouvoir de marché pour une banque sur ces anciens clients. Une firme qui est «capturée ${ }^{6}$ » en termes d'information par sa banque continue à entretenir des relations avec elle. En deuxième période, les banques installées ont un comportement de leader en prix sur leurs clients et les autres banques exercent une concurrence à la Bertrand. De fait, il est très difficile pour une firme peu risquée de persuader les autres banques qu'elle est effectivement de faible risque, car seule sa banque possède une bonne information sur ce risque. Cependant, la présence des phénomènes de

5. Terme utilisé par Sharpe, 1990.

6. Ibidem. 
sélection adverse et de risque moral au marché du crédit ne permet pas aux prêteurs de discriminer parfaitement les emprunteurs. Cela s'explique par le fait que la banque ne peut pas offrir aux clients d'une autre banque les mêmes conditions de prêt (même taux d'intérêt) qu'elle offre à ses clients.

Ce qui découle de l'analyse faite par Sharpe, en 1990, est l'existence d'une certaine segmentation du marché du crédit en fonction de la taille des entreprises. Pour les grandes entreprises, où une information relativement fiable (rating) est disponible pour toutes les banques présentes sur ce marché, l'information dont dispose la banque qui finance ce type d'entreprises ne diffère pas beaucoup de celle que possèdent les autres banques. Dans ce cas, le pouvoir de marché créé par les relations de clientèle est faible et l'inefficience dans l'allocation du capital prêté par les banques sera réduite, voire annulée, puisque l'équilibre du marché du crédit se rapproche de l'équilibre concurrentiel. Néanmoins, dans le cadre des PME où l'information sur la qualité de ces entreprises n'est ni précise ni particulièrement fiable, la banque qui finance ces entreprises possède des informations supplémentaires par rapport aux autres banques. Donc, dans le cas des PME, les banques ont un certain pouvoir de monopole sur leurs anciens clients, et il en résulte une inefficience de l'allocation du capital.

En effet, le volume d'informations publiques (billets de trésorerie, évaluations) est plus limité pour les PME que pour les grandes entreprises, ce qui explique le fait que les banques peuvent exploiter cette rente informationnelle en appliquant des taux débiteurs plus élevés.

En conséquence, nous sommes en présence d'un marché du crédit segmenté selon la taille des emprunteurs: un marché du crédit aux grandes entreprises très concurrentiel et efficient et un marché du crédit aux entreprises de dimension petite et moyenne moins concurrentiel et donc moins efficient dans la mesure où, sur ce marché, les banques peuvent retirer des profits de leurs anciens clients.

Ainsi, on retrouve la thèse suivante: les PME se financent plutôt par les banques locales ou régionales (Dietsch, 1993; Martin, 1995), tandis que les grandes entreprises se financent par des établissements bancaires d'envergure nationale ou internationale.

En effet, ce résultat se confirme sur le plan empirique. D'après les travaux de la Centrale des Bilans de la Banque de France ${ }^{7}$, la dispersion des taux entre grandes entreprises et PME est d'autant plus faible que l'entreprise est saine (tableau 2).

7. CNC (1988), « Rapport sur le coût du crédit selon la taille des entreprises », p. 49. 


\section{TABLEAU 2}

Coût apparent*

de l'endettement et classes de risques

(en \%)

\begin{tabular}{lccc}
\hline Classes de risques ** & PME (1) & Grandes entreprises (2) & Écart (1)-(2) \\
\hline Très défavorable & 13,4 & 9,8 & 3,6 \\
Défavorable & 13,4 & 1,3 & 3,1 \\
Moins défavorable & 14,1 & 10,8 & 3,3 \\
Neutre & 14,0 & 10,4 & 3,6 \\
Favorable & 13,2 & 10,9 & 2,3 \\
Plus favorable & 12,0 & 10,4 & 1,6 \\
Très favorable & 10,6 & 9,9 & 0,7 \\
\hline
\end{tabular}

* Médiane des taux.

** Fonction « $Z$ » de la Centrale de Bilans de la Banque de France.

Source: Centrale de Bilans de la Banque de France (1988).

La répercussion du risque dans les taux d'intérêt apparent semble donc plus marquée pour les PME que pour les grandes entreprises, ce qui s'explique sans doute à la fois par une meilleure capacité de négociation et par des possibilités d'accès à une gamme de financements moins onéreux.

Cette inefficience dans l'allocation du capital qui engendre les relations de clientèle peut être réduite par l'introduction des contrats implicites (Fried et Howitt, 1980). Dans ce cadre, la banque peut introduire une "promesse» spécifiant le taux pratiqué pour la période suivante contingent au résultat de la firme en première période.

Les arguments développés précédemment rejoignent la thèse de Perrien et Ricart (1994), où les relations de clientèle sont caractérisées de la façon suivante: «elles s'inscrivent dans une perspective de long terme, basée sur la connaissance mutuelle des deux partenaires, fondée sur une certaine forme de personnalisation et de confiance partagée, et porteuse de bénéfices mutuels » (p. 22).

Toutes ces conclusions théoriques se confirment sur le plan empirique. En effet, selon l'étude de Dietsch (1989), les effets de clientèle et de marchandage de taux permettent aux PME d'accéder à des modes de financement plus souples, mieux adaptés à leurs besoins et cela, à un coût moins élevé. En effet, les relations de clientèle auront des conséquences tout autant sur la disponibilité du crédit que sur son coût. Ainsi, l'augmentation de la qualité et de la quantité d'information qui résulte tend à réduire les bases du rationnement du crédit. Cela vient de ce que la banque, disposant d'une information plus précise, accroît l'efficacité de ses opérations d'évaluation du risque et, par voie de 
conséquence, diminue son degré d'exposition au risque de crédit. Cette plus grande efficacité dans la mesure du risque contribue également à réduire le coût du crédit, non seulement parce qu'elle aboutit à une tarification plus efficiente du crédit, mais également parce qu'elle tend à en réduire les coûts opératoires de gestion, notamment ceux occasionnés par les opérations de recherche d'information, de contrôle et de surveillance des crédits.

Pour les PME, les avantages à attendre, à travers l'amélioration de leur capacité de négociation, d'une baisse des taux et d'un meilleur accès au crédit sont sensibles.

Toutefois, la bonne négociation des conditions de crédit suppose que le banquier soit perçu comme un partenaire, ce qui implique que l'entreprise puisse mettre régulièrement à sa disposition des éléments comptables et prévisionnels fiables, permettant une relation «équilibrée» (à défaut, les financements seront orientés vers les ressources courtes et peu risquées). En effet, selon Dietsch (1989), ce sont les entreprises les mieux disposées à présenter des documents prévisionnels et à expliquer leur stratégie aux banques qui obtiennent les meilleures conditions; en outre, les PME qui pensent que les banques surestiment leur risque se rencontrent parmi celles qui recourent de façon moins systématique aux outils de gestion de trésorerie et transmettent moins d'informations aux banques.

\section{Conclusion}

La question centrale posée lors du financement des entreprises et qui a joué un rôle central tout au long de ce travail est celle de la qualité de l'entrepreneur. La qualité des emprunteurs qui se distinguent en «bons» et en «mauvais», selon le risque d'insolvabilité ou de défaut qu'ils représentent, fait que certains d'entre eux ne seront pas financés.

L'objectif de cet article était d'exposer les avancées théoriques qui se fondent sur cette problématique pour justifier l'éviction que subissent les PME en matière de financement. Les études empiriques mettent en évidence les caractéristiques économiques de ces entreprises, et nous avons cherché à saisir, en choisissant des modèles pionniers qui ont expliqué le phénomène de rationnement du crédit, les raisons pour lesquelles les PME se trouvent dans l'impossibilité de se financer.

Toutefois, le recours au rationnement arbitraire auquel font référence ces analyses tient compte uniquement des faits objectifs que sont les probabilités de défaut de l'emprunteur.

Les relations de clientèle permettent, comme nous venons de le voir, un meilleur traitement de l'incertitude du point de vue du prêteur et de l'emprunteur, 
puisqu'elles conduisent à diminuer les effets de sélection adverse et de risque moral et, par conséquent, le rationnement. En effet, le degré de rationnement du crédit par les banques peut être supposé inversement lié à la «qualité» des relations de clientèle. Le choix d'investissement de l'entrepreneur est souvent conditionné par le fait que le financement sera renouvelé. Ces relations de clientèle tendent, par conséquent, à rendre l'information entre prêteurs et emprunteurs symétrique.

Dans ce cas, la relation de crédit se fonde sur la répétition; elle devient personnalisée en tenant compte des spécificités de chaque entrepreneur. Ainsi, l'appréhension de l'incertitude quant à la qualité de l'entrepreneur ne se réduit pas au simple calcul statistique mais prend en compte d'autres éléments qui sont beaucoup plus subjectifs.

Par ailleurs, dans cet article, nous avons mis en évidence que la coordination entre les acteurs (prêteurs et emprunteurs) ne passe pas nécessairement par les prix, mais plutôt par d'autres moyens fondés sur la confiance, sur les relations durables et de long terme qui permettent de prendre en compte la singularité des individus et réduire ainsi l'incertitude des deux acteurs.

Donc, les banques ne se trouvent pas limitées par une stratégie d'octroi des fonds prêtables dont la seule dimension serait le prix des ressources prêtables, mais possèdent une marge de manœuvre beaucoup plus large.

Par conséquent, les relations de clientèle peuvent jouer un rôle fondamental dans l'octroi de prêts aux PME, la confiance accordée par le prêteur au producteur pouvant apparaître comme un substitut à l'analyse financière, notamment en raison du caractère souvent peu formalisé des documents comptables présentés par les dirigeants d'unités de petite dimension (Levratto, 1990).

Dans cette optique, dès que les PME affirment leur capacité de négociation en développant les outils de prévision et de suivi de la trésorerie, elles sont en mesure de tirer parti de la précision de l'information (ce qui permet une bonne évaluation du risque par la banque, donc des meilleures conditions pour l'entreprise), de la mise en concurrence des banques et du contrôle des conditions bancaires.

Ainsi, les banques doivent devenir les interlocuteurs privilégiés des dirigeants de PME. Cette coopération ne doit pas uniquement concerner les prêts accordés à l'entreprise, mais également la gestion générale de la firme (Levratto, 1990). La relation banque-entreprise prendrait ainsi une nouvelle dimension qui permettrait de dépasser les conflits latents ou ouverts souvent fréquents entre prêteurs et emprunteurs. Donc, il faut offrir aux PME un partenaire financier qui les accompagne dans leur développement, un peu à l'exemple des «Hausbanks» allemandes. 


\section{Bibliographie}

AFB (1994), «La banque et l'entreprise», $1^{\text {er }}$ Congrès de l'Association Française des Banques, 29 septembre, Paris, Carrousel du Louvre.

AKERLOF, G. (1970), «The markets for lemons: quality uncertainty and the market mechanism », The Quarterly Journal of Economics, vol. 84, août, p. 488-500.

BARDos, M. (1990), «Le crédit plus cher pour les petites entreprises», Économie et Statistique, $\mathrm{n}^{\mathrm{0}} 236$, octobre.

BARdos, M. (1991), «Méthodes des scores de la Centrale de Bilans », Document Banque de France, Centrale de Bilans, septembre, $65 \mathrm{p}$.

Belletante, B. (1991), «Pour une approche des spécificités financières de la PME au travers du concept de territoire financier», Revue Internationale PME, vol. $4, n^{0} 1$, p. 49-80.

Bloch, L., J. Bourdieu, B. Colin-Sedillot et G. Longueville (1995), «Du défaut de paiement au dépôt de bilan: les banquiers face aux PME en difficulté», Revue d'Économie Financière, n $^{\circ} 32$, printemps, p. 229-256.

CONSEIL NATIONAL DU CRÉDIT (1988), «Le coût du crédit aux entreprise selon leur taille», Bulletin du Crédit National, $4^{\mathfrak{e}}$ trimestre.

Cukierman, A. (1978), "The horizontal integration of the banking firm, credit rationing and monetary policy », Review of Economics Studies, vol. 45, $\mathrm{n}^{\circ} 1$, février, p. 165-178.

DiAmond, D. (1984), «Financial intermediation and delegated monitoring», Review of Economic Studies, vol. 51, n 3, p. 393-414.

DietsCh, M. (1989), «Les PME et les conditions bancaires», Revue d'Économie Financière, $\mathrm{n}^{\circ} 10$, juillet-septembre, p. 72-85.

DIETSCH, M. (1993), «L'organisation de l'industrie bancaire: les apports de la théorie bancaire», Contribution à la $8^{\mathrm{e}}$ session d'école d'été méditerranéenne d'économie industrielle, Cargèse, 20-25 septembre.

FRIED, J. et P. HowITT (1980), «Credit rationing and implicit contract theory», Journal of Money Credit and Banking, vol. 12, $\mathrm{n}^{0} 3$, p. 471-487.

Gale, D. et M. Hellwig (1985), «Incentive-compatible debt contracts: the one period problem», Review of Economic Studies, vol. 52, nº 4, p. 647-663.

HAUBRICH, J. (1989), «Financial intermediation, delegated monitoring and long-term relationships ", Journal of Banking and Finance, $\mathrm{n}^{\circ} 13$, p. 9-20.

JULIEN, P.A. (1994), Les PME: bilan et perspectives, Québec, Les Presses Inter Universitaires; Paris, Economica.

Levratto, N. (1990), «Le financement des PME par les banques: contraintes des firmes et limites de la coopération», Revue Internationale PME, vol. $3, \mathrm{n}^{\circ} 2$, p. 192-213. 
LEVRATto, N. (1992), «Une analyse du statut théorique de la notion de rationnement du crédit », dans R. Arena et D. Torre (éd.), Keynes et les nouveaux keynésiens, Paris, Presses universitaires de France, p. 333-352.

MARTIN, F. (1995), «Concurrence bancaire et asymétrie d'information», Contribution aux XII Journées internationales d'économie monétaire et bancaire, Nancy, $15-16$ juin.

Mattesini, F. (1993), Financial Markets, Asymmetric Information and Macroeconomic Equilibrium, Dartmouth.

MILDE, H. et J.G. RILEY (1988), «Signaling in credit markets», The Quarterly Journal of Economics, vol. CIII, $\mathrm{n}^{\circ}$ 1, février, p. 101-130.

OKUn, A.M. (1981), Prices and Quantities: A Macroeconomic Analysis, Washington, The Brookings Institution.

PARANQue, B. (1992), «L'enjeu du financement des PMI», Banque de France, Bulletin trimestriel, $n^{\circ} 84$, décembre.

PERRIEN, J. et L. RICARD (1994), «L'approche relationnelle dans le secteur bancaire», Revue Internationale de Gestion, vol. 19, no 4, p. 21-26.

PSILlAKI, M. (1992), «Le financement des PME», Mémoire de DEA, Université de Nice-Sophia-Antipolis.

RILEY, J.G. (1987), «Credit rationing: a further remark», The American Economic Review, vol. 77, $\mathrm{n}^{\circ} 1$, mai, p. 224-227.

RIVAud-DANSET, D. (1995), «Le rationnement du crédit et l'incertitude», Revue d'Économie Politique, $\mathrm{n}^{\mathrm{0}} 2,105^{\mathrm{e}}$ année, mars-avril, p. 223-247.

Rivaud-Danset, D. et R. SAlais (1992), «Les conventions de financement des entreprises », Revue Française d'Économie, vol. VII, nº 4, automne, p. 81-120.

Roger-Machart, J. (1991), Réussir nos PME, Paris, Dunod.

SHARPE, S. (1990), «Asymmetric information, bank lending, and implicit contracts: a stylised model of customer relationships », Journal of Finance, septembre, p. 1069-1087.

STIGLITZ, J.E. (1987), «The causes and consequences of the dependence of the quality on price», Journal of Economic Literature, vol. XXV, mars, p. 1-48.

STIGLITZ, J.E. et A. WEISS (1981), «Credit rationing in markets with imperfect information», The American Economic Review, vol. 71, n 3, juin, p. 393-410.

STIGLITZ, J.E. et A. WEISS (1983), «Incentive effects of terminations : applications to the credit market and labor markets", The American Economic Review, vol. $73, \mathrm{n}^{0} 5$, décembre, p. 912-927.

StiglitZ, J.E. et A. Weiss (1987), «Credit rationing: reply», The American Economic Review, vol. 77, no 1, mai, p. 228-231. 
WEBB, D. (1992), «Two period financial contracts with private information and costly state verification », Quarterly Journal of Economics, vol. CVII, août, p. 1113-1123.

YonCOURT, B. et T. MAROIS (1990), «Le financement des PME par les banques», Revue d'Économie Financière, ${ }^{\text {os }}$ 12-13, printemps-été, p. 27-40. 